\title{
Le braconnage et la population d'éléphants du parc national de Zakouma (Tchad)
}

\section{Pierre PoILeCot}

Centre de coopération internationale en recherche agronomique pour le développement (Cirad) Département Environnements et Sociétés Campus international de Baillarguet 34398 Montpellier Cedex 5 France

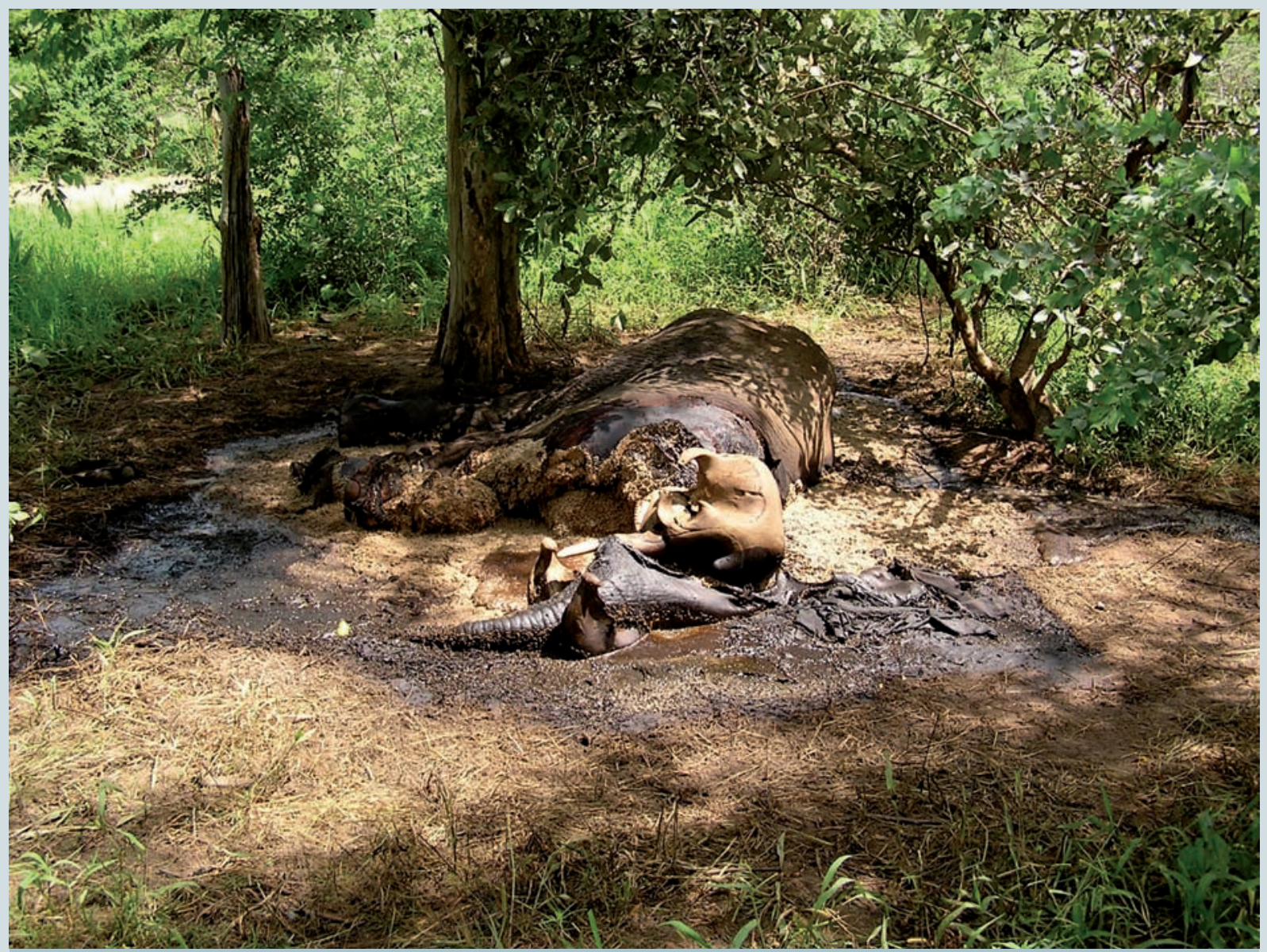

Photo 1.

Cadavre d'éléphant en décomposition, les défenses ayant été arrachées par les braconniers. Photo P. Poilecot. 


\section{RÉSUMÉ}

\section{LE BRACONNAGE ET LA POPULATION D'ÉLÉPHANTS DU PARC NATIONAL DE ZAKOUMA (TCHAD)}

En 2005, le parc national de Zakouma, dans le sud-est du Tchad, abritait une population de 3900 éléphants, correspondant à une densité exceptionnelle proche de 1,3 individu par kilomètre carré au sein des aires protégées d'Afrique francophone. Les résultats des dénombrements aériens réalisés en 2006 ont révélé une diminution de $22 \%$ des effectifs de l'éléphant. Le déclin de la population s'est ensuite accentué brutalement, de 940 individus en 2008 à seulement 620 en 2009. Les activités de braconnage, d'une rare intensité en 2008 , ont conduit à éradiquer près de $70 \%$ de la population d'éléphants du parc sur une période de cinq ans. Les informations relatives au braconnage dans le parc de Zakouma rejoignent les récentes conclusions publiées par les programmes Mike et Etis, qui suivent l'évolution du braconnage et analysent les tendances du commerce illicite de l'ivoire dans les États membres de la Cites. La recrudescence du braconnage correspond à la reprise du commerce international de l'ivoire en 2006, avec une forte augmentation des prix consécutive à une demande accrue de la part de certains pays asiatiques. L'ivoire collecté en Afrique centrale est intégré au trafic à partir de réseaux installés au Soudan, de mieux en mieux structurés, et les éléphants de Zakouma, ne portant pourtant que de petites défenses, ne sont pas épargnés. La recherche scientifique, à partir de la définition des empreintes génétiques des éléphants, qui permet de déterminer la provenance géographique de l'ivoire, et le suivi du braconnage par les programmes Mike et Etis constituent des éléments majeurs pour mieux connaître et suivre l'évolution des populations d'éléphants. Cependant, la mauvaise "gouvernance » des États, en particulier en termes de politique de conservation et de stratégie de lutte anti-braconnage, demeure le facteur essentiel de la recrudescence du braconnage de l'éléphant.
ABSTRACT

\section{POACHING AND THE ELEPHANT POPULATION IN ZAKOUMA NATIONAL PARK, CHAD}

In 2005, Zakouma National Park, in south-eastern Chad, had a population of 3900 elephants, an exceptionally high density for the protected areas of French speaking Africa, of nearly 1.3 individuals per square kilometre. However, results from aerial population counts carried out in 2006 showed a $22 \%$ drop in elephant numbers. The decline then suddenly accelerated, with 940 individuals in 2008 dropping to just 620 in 2009. Poaching, which increased to record levels in 2008 , had destroyed almost $70 \%$ of the elephant population in the park in five years. Information on poaching in the Zakouma Park agrees with recent conclusions published by the MIKE and ETIS programmes, which are monitoring the rise of poaching and analyzing trends in the illegal ivory trade in CITES member countries. The upsurge in poaching coincides with the reopening of the international ivory trade in 2006, which brought a steep increase in prices following increased demand from some Asian countries. Ivory from central Africa finds its way onto the black market through increasingly well organized networks established in Sudan, including from Zakouma's elephants despite the relatively small size of their tusks. Scientific research, through genetic footprints defined for the elephants, with which the geographical origin of the ivory can be determined, and monitoring of poaching by the MIKE and ETIS programmes, are crucial to a better understanding of elephant populations and how they are evolving. However, inadequate governance in the states concerned, especially in terms of conservation policies and anti-poaching strategies, remains the key factor in the current upsurge in elephant poaching.

Keywords: elephant, poaching, ivory trade, Zakouma, Chad.
RESUMEN

\section{EL FURTIVISMO Y LA POBLACIÓN DE ELEFANTES DEL PARQUE NACIONAL DE ZAKOUMA - CHAD}

En 2005 el Parque Nacional de Zakouma, en el sudeste de Chad, albergaba una población de 3900 elefantes. Esto suponía una densidad excepcional, próxima a 1,3 individuos por kilómetro cuadrado, dentro de las áreas protegidas del África francófona. Los resultados de los censos aéreos realizados en 2006 revelaron una disminución del $22 \%$ de la población de elefantes. El declive de la población se aceleró luego de forma brutal, pasando de 940 individuos en 2008 a únicamente 620 en 2009. Las actividades de furtivismo, de una rara intensidad en 2008, provocaron la desaparición del $70 \%$ de la población de elefantes del parque en un período de cinco años. Las informaciones sobre el furtivismo en el Parque de Zakouma confirman las recientes conclusiones publicadas por los programas MIKE y ETIS, que siguen la evolución del furtivismo y analizan las tendencias del comercio ilícito de marfil en los estados miembros de la CITES. El recrudecimiento del furtivismo se corresponde con la reanudación del comercio ilegal de marfil en 2006, con un fuerte incremento de precios provocado por la creciente demanda de algunos países asiáticos. El marfil recolectado en África Central se integra en el tráfico a partir de redes implantadas en Sudán, cada vez mejor estructuradas; y los elefantes de Zakouma, a pesar de que sólo llevan colmillos pequeños, no se libran de la amenaza. La investigación científica, a partir de las huellas genéticas de los elefantes, que permiten determinar el origen geográfico del marfil, y el seguimiento del furtivismo, a través de los programas MIKE y ETIS, constituyen elementos indispensables para conocer mejor y monitorear la evolución de la población de elefantes. Sin embargo, la mala gobernanza de los estados, especialmente en cuanto a políticas de conservación y de estrategia de lucha contra el furtivismo, sigue siendo el factor esencial del recrudecimiento de la caza furtiva de elefantes.

Palabras clave: elefante, caza furtiva, comercio de marfil, Zakouma, Chad.

commerce de l'ivoire, Zakouma, Tchad. 


\section{Introduction}

Les mesures de conservation mises en œuvre dans le parc national de Zakouma, entre 1989 et 2005 , ont permis une reconstitution spectaculaire des populations de grands mammifères (ARRANz et al., 2007 ; PoIlecot, 2010). En effet, dans les années 1970-1980, cette aire protégée, située dans le sud-est du Tchad (figure 1), avait souffert des conséquences de conflits armés, de sécheresses récurrentes et d'une épidémie de peste bovine qui en avaient sévèrement affecté le patrimoine faunique. Dès 1989, la volonté du gouvernement tchadien et le soutien de l'Union européenne ont conduit à réhabiliter ce parc qui constitue actuellement un véritable sanctuaire de $3000 \mathrm{~km}^{2}$ pour la faune soudanienne de ce pays.

Si le rhinocéros noir a disparu du Tchad, alors que le parc en abritait encore une dizaine en 1968 (DEJACE, 2002), d'autres espèces emblématiques demeurent à Zakouma. Parmi elles, les principales restant dans le parc sont l'éléphant (Loxodonta africana), la girafe (Giraffa camelopardalis antiquorum), le guépard (Acinonyx jubatus) et le lycaon (Lycaon pictus, récemment observé sur la bordure ouest du parc). Les savanes soudano-sahéliennes, principalement constituées de peuplements d'Acacia seyal ou de combrétacées, supportent de très belles populations d'ongulés et de carnivores qui sont exceptionnelles dans une aire protégée d'Afrique francophone.

Les inondations annuelles, qui recouvrent en partie le parc de Zakouma entre juillet et octobre, sont un élément essentiel de l'écosystème. En effet, elles permettent d'alimenter en eau et en limons de vastes dépressions herbeuses qui procurent un pâturage d'une grande qualité à la faune tout au long de la saison sèche, au fur et à mesure du retrait de l'eau.

Ces inondations, qui réduisent l'espace vital des grands mammifères, obligent certaines espèces comme l'éléphant, le damalisque

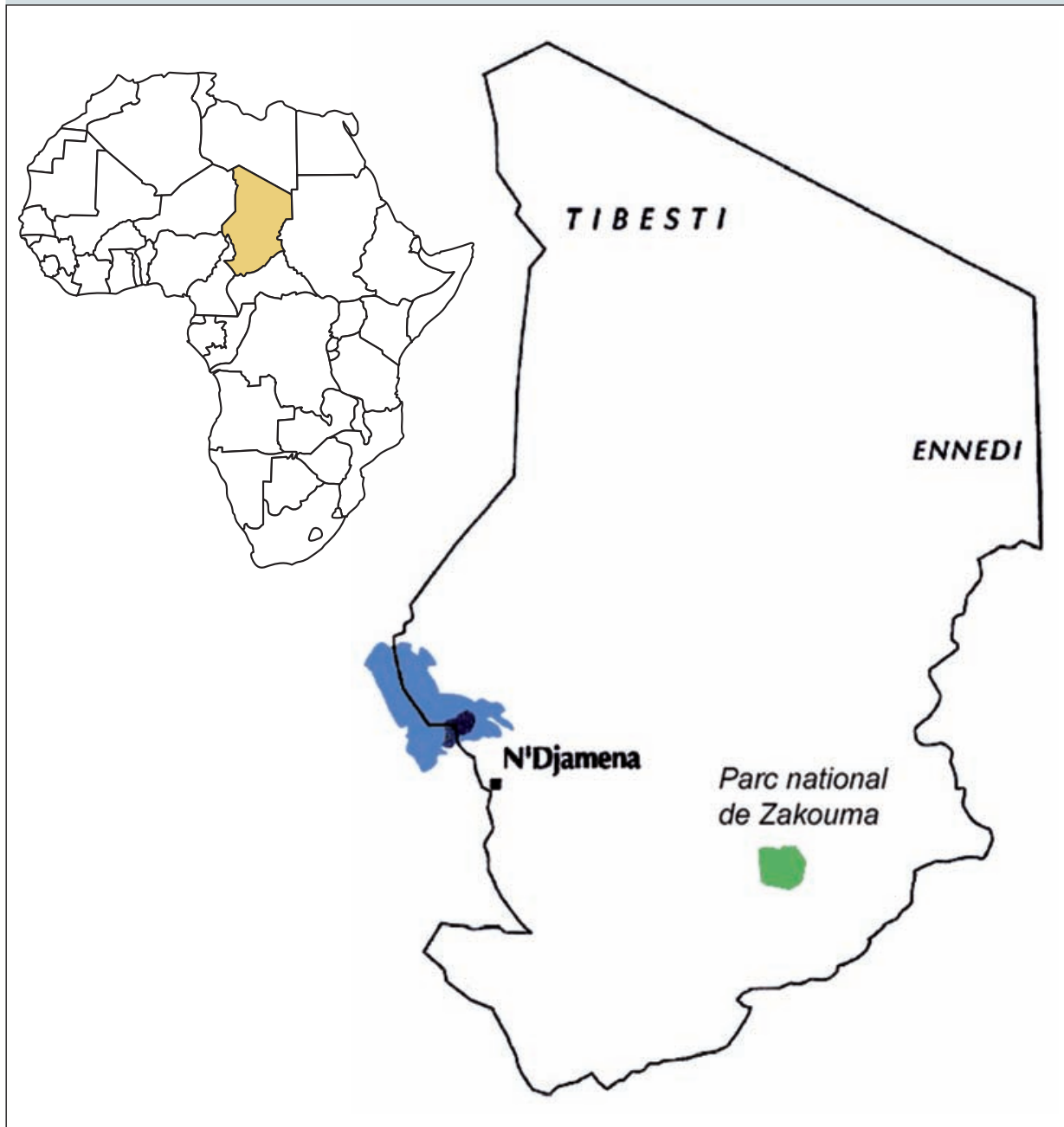

Figure 1.

Situation du parc national de Zakouma au Tchad.

(Damaliscus lunatus tiang), le bubale (Alcelaphus buselaphus lelwel) et l'hippotrague (Hippotragus equinus) à migrer en dehors du parc (DOLMIA, 2004 ; DolmiA et al., 2007 ; FAY et al., 2006b ; POILECOT, 2008). Ces déplacements saisonniers sont les plus spectaculaires pour l'éléphant, observé à plus de cent kilomètres des limites du parc, et le damalisque dont la population entière quitte l'aire protégée jusqu'aux confins de la zone sahélienne.

Si la succession des campagnes de dénombrements aériens des grands mammifères au cours de la saison sèche, mise en œuvre depuis 1986 (BOUSQUET, 1986, 1991 ; DEJACE et al., 2000 ; MACKIE, 2002 ; FAY et al.,
2005, 2006a), a permis de constater une progression ou parfois la stabilité des populations animales, elle a révélé dès 2006 une diminution de la population d'éléphants (FAY et al., 2006a et 2006b). En effet, estimée à près de 3900 animaux en 2005, elle ne comptait plus que 3000 individus en 2006, indiquant une différence de $22 \%$ dans les effectifs. Des comptages plus récents (POILECOT, 2008 ; Potgieter et al., 2009) ont alarmé les gestionnaires de l'aire protégée puisque la population fut estimée à seulement 937 éléphants (dénombrement aérien par échantillonnage systématique) en 2008 et 620 en 2009 (dénombrement aérien total). 


\section{Le braconnage : un problème à l'échelle du continent africain}

Le graphique présenté dans la Figure 2.

Évolution des effectifs d'éléphants et des carcasses observée entre 2002 et 2009 (les carcasses correspondent à des carcasses « fraîches » et " récentes »). 2002, 2008 : comptage par échantillonnage systématique ; 2006, 2008, 2009 : comptage total ; $2006^{*}$ : dénombrement de saison des pluies.

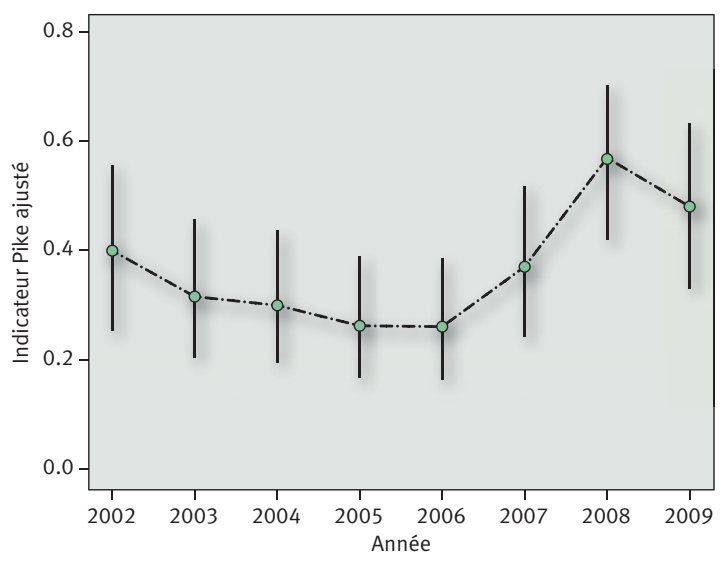

Figure 3.

Évolution de l'indicateur Pike d'après les informations obtenues en Asie et en Afrique pour la période 2002-2009 (source : Cites, CoP15 Doc 44.1, 2010).

\section{Une augmentation du nombre de carcasses}

À cette diminution drastique des effectifs des éléphants, sur une période de trois années, est corrélée une augmentation des carcasses « fraîches » et « récentes » observées au cours des dénombrements aériens (figure 2).

Les activités de braconnage observées en 2002 ont considérablement régressé par la suite pour reprendre en 2006, où elles ont été détectées à la suite d'un dénombrement de saison des pluies qui avait conduit à enregistrer un nombre important de cadavres fraîchement abattus tant dans le parc que dans la zone périphérique (photo 1 ).
Le nombre important de cadavres enregistré en 2008 correspond à une estimation à l'échelle du parc, à partir de 124 carcasses effectivement observées lors du comptage. La chasse à l'éléphant régresse en 2009 et la mise à disposition d'un avion par la Wildlife Conservation Society (WCS) a permis d'intensifier les patrouilles aériennes qui semblent avoir eu un effet dissuasif sur les braconniers. À cette augmentation du nombre de carcasses correspond également un changement dans la distribution de celles-ci. En effet, les récents dénombrements indiquent que la majeure partie des cadavres se situe dans la partie est du parc qui abrite le réseau hydrographique et assure ainsi des ressources permanentes en eau aux animaux. De plus, un nombre important de carcasses a été cartographié dans les environs du poste des gardes de Zakouma et du campement touristique de Tinga (PoILECOT et al., 2010, sous presse). figure 2 est conforme aux informations (indicateur Pike : Proportion of Illegally Killed Elephants) obtenues au travers du programme Mike (Monitoring the Illegal Killing of Elephants) pour le parc de Zakouma et rapportées dans le document de préparation de la Quinzième Conférence des Parties de la Cites qui doit se tenir à Doha (Qatar) au mois de mars 2010 (CITES, 2010). Le programme Mike, permettant de suivre l'évolution du braconnage dans les différents États membres de la Cites, a été mis en place en Afrique de l'Ouest, centrale et de l'Est en 2002.

Ce programme qui couvre $16 \%$ de la superficie des territoires occupée par l'éléphant doit fournir, au travers de la Cites, un rapport actualisé sur les informations collectées sur le braconnage à chacune des Conférences des Parties. C'est ainsi que Mike a pu analyser l'évolution du braconnage en Afrique à partir de données concernant 2316 carcasses d'éléphants obtenues dans 47 sites Mike pendant la période 2002-2008. L'évolution de l'indicateur Pike pour l'Asie et l'Afrique sur une période de huit ans (figure 3) confirme les résultats présentés dans la figure 2. Le braconnage qui avait diminué à partir de 2002 reprend dès 2006 pour atteindre un pic en 2008. Le déclin en 2009 doit être considéré avec réserve du fait d'un manque de données, en particulier en provenance d'Afrique centrale qui est la région avec le plus fort indicateur Pike.

Le problème du braconnage apparaît donc général à l'échelle du continent. Quatre facteurs principaux sont mis en avant pour appréhender l'intensité du braconnage : le type d'écosystème considéré, la protection mise en œuvre, la facilité d'accès au milieu pour l'homme (pistes) et le Corruption Perception Index (Cpi). Les conclusions des études entreprises par le programme Mike mettent en évidence que l'intensité de braconnage est : 


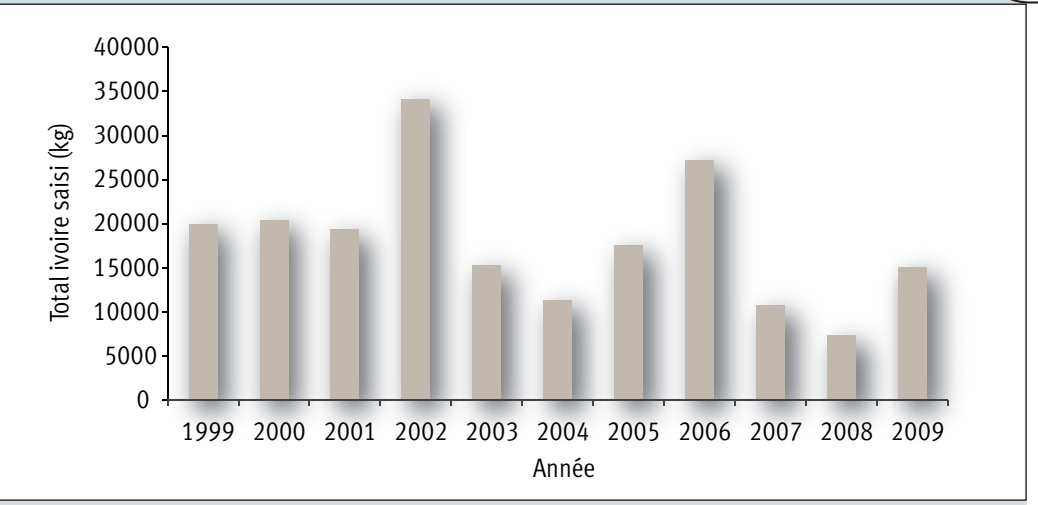

Figure 4.

Estimations (en kg) des volumes d'ivoire brut saisis pour la période 1999-2009 (source : MILIKEN et al., 2009).

- la plus réduite dans les États assurant une « gouvernance » efficace, en particulier dans la mise en œuvre et le respect des politiques de conservation ; - réduite dans les sites couvrant de vastes superficies (difficulté de localiser les éléphants, logistique plus lourde à mettre en place par les braconniers...) ; - plus forte dans les écosystèmes forestiers, dont le couvert végétal est le plus dense (facilité d'approche des animaux, contrôle des patrouilles de surveillance moins efficace); l'Afrique centrale est considérée comme ayant le plus fort niveau de braconnage avec $63 \%$ des carcasses enregistrées provenant d'éléphants illégalement tués ;

- plus forte dans les sites abritant d'importantes populations d'éléphants.

\section{L’écueil}

En matière de protection de l'environnement et de la biodiversité, le Tchad est signataire des principales conventions internationales et régionales. Malgré cela, le milieu naturel et les aires protégées demeurent soumis à de fortes pressions anthropiques occasionnées par l'élevage, l'agriculture, l'utilisation non durable des ressources naturelles, une mauvaise gestion des feux de brousse et le braconnage (de mieux en mieux organisé). Le contexte politique global du Tchad est défavorable à la conservation de la nature, avec de plus le conflit du Darfour qui vient exacerber les problèmes intérieurs du pays.

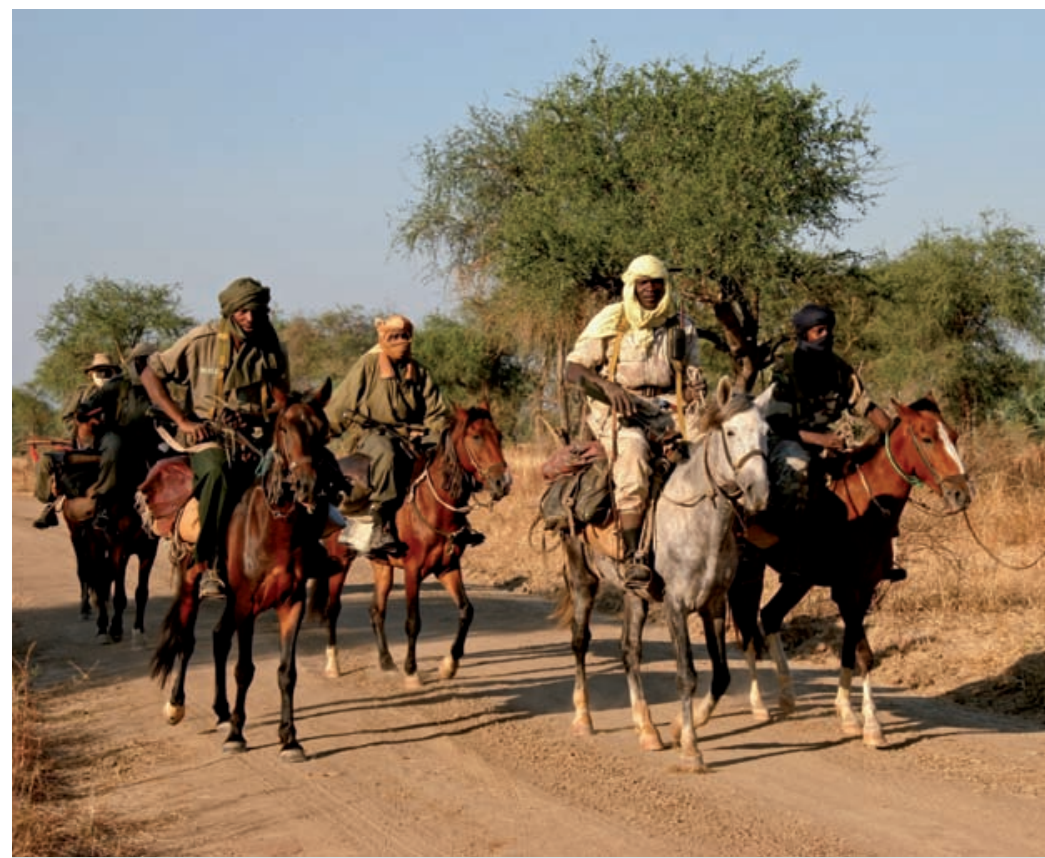

Photo 2.

Patrouille de gardes à cheval.

Photo P. Poilecot.

Le parc de Zakouma, contrairement à de nombreuses autres aires protégées, a bénéficié d'un appui conséquent de l'Union européenne depuis 1989 et est doté, depuis 2008, d'un plan de gestion. II est d'un accès facile (en véhicule, à cheval ou à pied) et abritait, jusqu'en 2005, une population importante d'éléphants avec 1,3 individu au kilomètre carré : cette densité atteint près de deux éléphants dans la partie est de l'aire protégée au cours de la saison sèche. En 2005, dans le cadre du projet Curess (Conservation et utilisation rationnelle des écosystèmes soudano-sahéliens), le volet Protection/Surveillance employait près de cent agents, soit un garde pour $30 \mathrm{~km}^{2}$ (photo 2). Ce déploiement de force constituait une exception pour les aires protégées d'Afrique francophone, pour lesquelles le personnel affecté à cette activité est souvent en nombre insuffisant.

La reprise inquiétante du braconnage dans le parc national de Zakouma, à partir de 2006, est imputable à divers facteurs intrinsèques : gouvernance inefficace en matière de conservation (au plan national), formation insuffisante des gardes, armement désuet de la cellule antibraconnage, activité de surveillance réduite au cours de la période de transition entre les phases I et II du projet Curess, entre 2006 et 2007.

D'autres facteurs extrinsèques et qui dépassent le cadre du Tchad sont également responsables de la recrudescence des tueries d'éléphants. Cet article n'a pas vocation à désigner les multiples acteurs impliqués dans le trafic d'espèces animales sauvages ou de produits animaux, qui sont par ailleurs médiatiquement clairement identifiés ; mais il ne fait aucun doute qu'ils sont à l'origine de la croissance des échanges économiques internationaux, engendrant une augmentation du cours de l'ivoire et une pression anthropique sur cette ressource naturelle. 
Photo 3.

Troupeau d'éléphants à l'abreuvoir : le poids des défenses,

même chez les animaux les plus gros, n'excède pas quelques kilogrammes. Photo P. Poilecot.

Le braconnage a diminué dans la plupart des États africains pendant les premières années qui ont suivi le moratoire sur le commerce de l'ivoire, mis en place en 1989, entraînant une chute du prix de l'ivoire jusqu'en 1996, mais ne dissipant pas pour autant les avertissements et inquiétudes de nombreuses associations de conservation et des gestionnaires de la faune en ce qui concerne les menaces pesant sur les populations d'éléphants.

Le système de contrôle Etis (Elephant Trade Information System), mis en place en parallèle de Mike en 1997, a pour objectif d'enregistrer et d'analyser les tendances du commerce illicite d'ivoire (et non de la chasse). Depuis 1989, cet organisme a collecté, auprès de 85 pays, près de 14500 informations relatives à des saisies d'ivoire. Le poids est un élément essentiel pour estimer l'impact du commerce de l'ivoire sur les populations d'éléphants. Or, les enregistrements des saisies d'ivoire concernent souvent un nombre de « pièces » sans information sur le poids. L'utilisation de modèles de régression permet de convertir ce nombre de pièces (ivoire brut, travaillé et semi-travaillé) en poids, en termes d'" équivalent en ivoire brut ». Environ 360 tonnes d'ivoire ont été saisies entre 1989 et
2009 dont 200 pour la période 1999 2009 (MILLIKEN et al., 2009) (figure 4). L'augmentation des saisies d'ivoire, liée à l'intensification des échanges avec notamment des pays d'Asie, a repris lors de la première vente unique d'ivoire à destination du Japon en 1999, puis s'est de nouveau accentuée par une seconde vente ayant eu lieu en 2007, à la suite de la levée partielle de l'interdiction du commerce de l'ivoire, permettant une vente vers l'Asie à partir de quatre pays d'Afrique australe.

Un total de 1414 défenses a été saisi à Taïwan, Hongkong et Osaka entre les 3 juillet et 28 août 2006, toutes d'origine africaine et en particulier de l'Est du continent (WASSER et al., 2009). En 2009, les autorités vietnamiennes se sont emparées d'une cargaison de 6,2 tonnes d'ivoire en provenance, de nouveau, d'Afrique orientale. Entre 25 et 29 tonnes d'ivoire, en provenance d'Afrique, auraient été confisquées au cours de l'année 2006, période à laquelle le braconnage reprend de façon intense au Tchad. En 2007, il fut également particulièrement sévère en République centrafricaine (CHARDONNET, BOULET, 2008). La répétition de ces évènements contredit le positionnement de certaines institutions de suivi des populations d'espèces sauvages : à titre d'exemple, la récente saisie de près de trois tonnes d'ivoire au Japon. Une grande partie de cet ivoire, provenant des dents les plus petites travaillées sur place en Afrique, est utilisée pour la confection de "hankos" qui sont des sceaux faisant office de signature (WASSER et al., 2009).

Dans certains pays, le prix de gros de l'ivoire a monté en flèche au cours des deux dernières décennies, passant de 100 dollars par kilogramme $(\$ / \mathrm{kg})$ à la fin des années 1990 à 750 dollars actuellement. Les pays développés de l'hémisphère Nord ne sont pas en marge de ce trafic (Stiles, Martin, 2009). La plupart des pays africains restent également des lieux privilégiés d'échange pour l'" or blanc » et abritent des « marchés d'ivoire » à ciel ouvert employant des milliers d'ivoiristes. D'après MARTIN (2005), les défenses travaillées au Soudan proviennent majoritairement de la République démocratique du Congo, de la République centrafricaine, du Tchad et du Kenya. Les douaniers considèrent que les saisies ne représentent pas plus de $10 \%$ du volume qui peut circuler dans le monde au travers de la contrebande : 250 tonnes d'ivoire (poids moyen des défenses de 6,6 kg) auraient donc été commercialisées au cours de l'année 2006. Corruption, falsification de documents, ateliers clandestins, réseaux de vente structurés permettant d'écouler des défenses ou des objets travaillés de contrebande dans le marché légal de l'ivoire : tout est parfaitement mis en œuvre pour soutenir et intensifier le commerce à l'échelle mondiale.

Le braconnage des éléphants au Tchad, vu la taille et le poids des défenses prélevées, pourrait laisser perplexe. La population d'éléphants du parc de Zakouma est jeune (photo 3) et ne comprend plus de " gros porteurs », ces éléphants possédant de longues et lourdes défenses.

L'ivoire obtenu sur chaque cadavre, même sur les animaux les plus vieux, ne représente que quelques kilogrammes (photo 4). Par 
BRACONNAGE DE L’ÉlÉPHANt / FORUM

Si la lutte armée, inévitable parfois, est un moyen de pression sur les braconniers, elle ne suffit pas à éliminer ou réduire le braconnage. Une lutte " préventive » est également indispensable et même préférable, à partir d'une présence continue des agents (renforcée si possible par un appui aérien), d'une collaboration avec les populations locales (informateurs), d'une communication soutenue (réseau) avec toutes les parties intéressées (instances politiques, administratives, locales et opérateurs privés, sociétés de safaris) et de l'éducation environnementale. Il s'agit donc de mettre en œuvre davantage une "lutte intelligente » plutôt que basée sur la force. La motivation des agents doit être favorisée et entretenue (salaires décents, primes, avantages en nature) par des moyens financiers conséquents. La lutte armée conduit à l'arrestation des braconniers, à la saisie des armes, des munitions et des moyens de transport (véhicules, chevaux, dromadaires...), mais souvent après l'abattage des éléphants (photo 5). La satisfaction d'avoir appréhendé les chasseurs, le matériel confisqué et l'ivoire récupéré pèsent peu face au nombre d'animaux abattus, avec tout ce que cela entraîne sur le plan de la conservation de l'espèce et du fonctionnement de l'écosystème.

Enfin, il existe un réel besoin de faire appliquer la loi dans le cadre de l'arrestation de braconniers (respect des procédures, législation sur l'utilisation des armes...) et de s'assurer que les condamnations découlant des délits sont effectivement appliquées. Trop de braconniers et de contrebandiers agissent en toute impunité : les peines encourues (emprisonnement, amendes) sont souvent dérisoires en comparaison du délit commis. Il y a également nécessité d'édifier les agents des parcs sur leurs réelles attributions et sur celles des autres membres des corps habillés. 


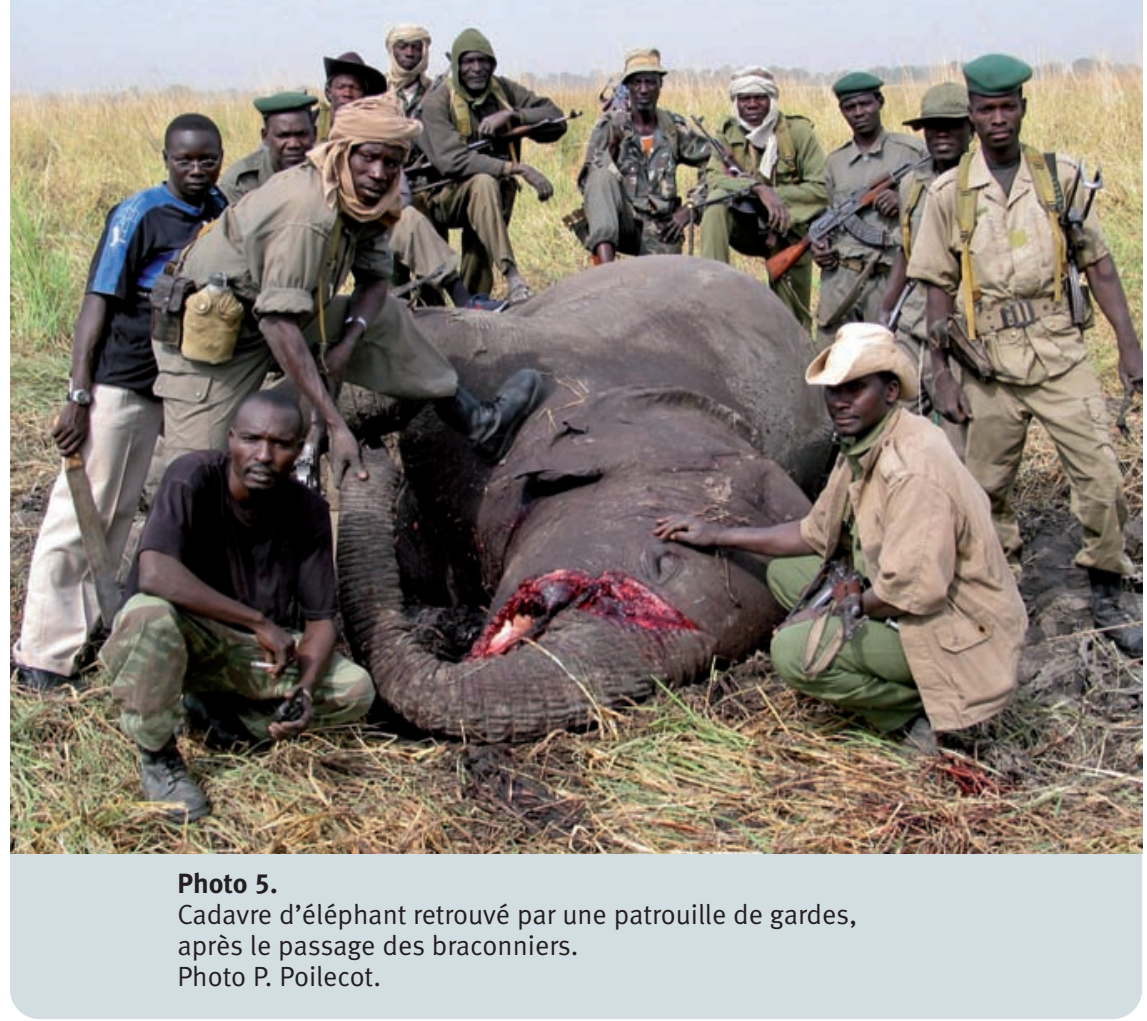

L'implication des braconniers dans le trafic de l'ivoire n'est pas le fait du hasard. Malgré la découverte récente de pétrole au Tchad, et sa mise en exploitation, la situation sociale des habitants ne semble pas s'améliorer. Ce pays est classé parmi les plus pauvres du monde et $64 \%$ de la population est en deçà du seuil de pauvreté national. Si les catastrophes naturelles - sécheresses, invasions acridiennes et d'oiseaux (mange-mil : Quelea quelea) - ont un impact certain, la chronicité de la malnutrition est principalement due à l'état de pauvreté du pays plutôt qu'à ces causes liées au déficit céréalier (NATIONS UNIES, 2005).

Le manque d'emplois, la famine liée à la pauvreté, une insécurité permanente tributaire de celle du Soudan et de la République centrafricaine sont autant de facteurs pour que se développent des activités illégales dans le cadre d'un processus de survie. Une personne qui tue des éléphants dans l'espoir d'un gain supplémentaire, en vendant de l'ivoire, s'impliquerait aussi bien dans le trafic du bois, des pierres pré- cieuses ou de toute autre matière première disponible et ayant une valeur commerciale.

Les braconniers constituent l'un des premiers maillons dans la filière de l'ivoire et certainement pas le plus lucratif. Ils ne portent, en fait, qu'une part minime de la responsabilité du trafic et ne sont que de simples exécutants. Ces hommes, appartenant à l'une des classes les plus démunies de la société, prennent des risques avec les agents des parcs en premier lieu, puis avec les autorités s'ils sont arrêtés, en considérant que la loi soit alors rigoureusement appliquée. Considérer le braconnier comme un « hors-la-loi » est une réalité, mais ne pas lui retirer le fait qu'il est avant tout un "homme " doit en être une autre. Ces chasseurs se mobilisent au départ pour tuer des animaux et non d'autres hommes. Il y a un besoin impérieux de ne pas oublier cette vérité pour éviter tout dérapage pouvant porter atteinte à ces populations et leurs familles.

À Zakouma, la lutte anti-braconnage est devenue une véritable guerre et chaque année des agents des parcs et des braconniers perdent la vie au cours des affrontements. Les premiers sont formés à la stratégie de lutte antibraconnage et au maniement des armes mais sont, pour la plupart, analphabètes. Cette formation, qui devrait également prendre en compte le rôle de la lutte anti-braconnage dans la conservation des espèces, le maintien de l'intégrité de l'aire protégée, les conséquences pour la recherche et le tourisme, et les répercussions environnementales et socio-économiques, fait davantage penser à un « apprentissage pour tuer ». L'attitude de certains agents en témoigne et seul le contact avec le métal de leur Kalachnikov semble un peu apaiser la peur qui les tenaille lors des patrouilles. Ces hommes sont mobilisables jour et nuit, avec des salaires qui ne récompensent pas, de très loin, leur dévouement et les risques qu'ils prennent dans leur travail. Les seconds sont des habitués de la " brousse » qui vivent en symbiose parfaite avec leur environnement. Ils survivent dans un dénuement des plus dramatiques, comme beaucoup de personnes dans les zones rurales, et sont donc prêts à tout pour gagner un minimum d'argent qui leur permettra un peu de mieux-être, pour eux-mêmes et leurs familles. Agents et braconniers sont des citoyens appartenant à la même ethnie ou à des ethnies voisines, qui parlent souvent la même langue, se connaissent même parfois et aspirent au même bien-être malgré une lutte fratricide. La lutte anti-braconnage a effectivement pour objectif premier de protéger des animaux, surtout s’ils sont menacés, rares ou emblématiques. Mais cette lutte ne doit pas conduire à la dérive précédemment évoquée entraînant l'élimination d'êtres humains. Est-il possible de considérer au même titre de «braconnier » un ramasseur de gomme arabique, un chasseur d'éléphants ou un pêcheur, tous il est vrai en situation illégale au sein d'une aire protégée ? Et doit-on, dans tous les cas, faire un usage abusif du pouvoir et des armes qui, très souvent, conduit à des incidents ou des accidents graves, regrettables et irréversibles? 


\section{En conclusion : quel espoir?}

L'extraction de l'Adn de fèces d'éléphants dans divers pays africains a permis de définir leurs empreintes génétiques. Il est ainsi possible de comparer cet Adn avec celui des défenses saisies et de déterminer leur provenance (WASSER et al., 2008). De tels résultats sont d'une grande importance pour mettre en œuvre des mesures de surveillance et de lutte anti-braconnage dans les pays où les éléphants sont les plus menacés. Cette nouvelle technologie représente un moyen de lutte préventive qui permet de situer géographiquement les inévitables futurs massacres. Il est ainsi possible de resituer le rôle du scientifique dans la lutte anti-braconnage tout en soulignant ses limites. En effet, la recherche dans un tel cadre, tant sur le suivi des populations d'éléphants que sur celui visant à définir la provenance géographique des défenses, fournit des résultats qui sont autant d'éléments de réflexion. Elle permet d'alerter, de recommander mais n'a pas de pouvoir politique dans les prises de décision.

La survie de l'éléphant à Zakouma, espèce emblématique du parc, ne tient qu'à la volonté de le conserver. Le gouvernement tchadien a un rôle majeur à jouer en mobilisant toutes les instances politiques, administratives et locales, les populations, et en collaborant avec toutes les parties intéressées ou impliquées dans la conservation. L'Union européenne, qui a tenu une place importante dans la réhabilitation du parc (et qui est toujours présente), ou tout autre bailleur, les Ong de conservation (internationales, nationales et locales), les projets de développement constituent autant d'acteurs qui peuvent agir et décider à différents titres, de manière individuelle ou concertée, pour permettre la conservation des écosystèmes et des espèces végétales et animales.
Le problème du commerce de l'ivoire reste posé alors que les réponses à de nombreuses questions pour le limiter sont connues, depuis toujours. Comment peut-on envisager l'arrêt du commerce de l'ivoire lorsque des "marchés de l'or blanc » s'affichent en toute impunité dans divers pays? Que deviennent le pouvoir et le rôle des grandes institutions de conservation, et directement des Nations unies, face à une contrebande dont les circuits sont parfois connus, tant au plan local qu'international ? Quel est le rôle et le poids des bailleurs de fonds, soutenant des projets de développement au sein desquels le braconnage est devenu une contrainte à leur bon fonctionnement et un facteur de risque pour le personnel employé ? Que doit-on penser des liens, au sein de ce trafic, avec les organisations criminelles impliquées dans le trafic des armes et des munitions?

Les récentes saisies d'ivoire brut ou travaillé, atteignant des volumes importants, témoignent d'un dysfonctionnement dans le système de contrôle et de suivi. MILLINKEN et al. (2009), au travers de l'organisme Etis, reconnaissent que peu de progrès ont été réalisés depuis la mise en place par la Cites, en 2007, du «Plan d'action pour le contrôle du commerce de l'ivoire de l'éléphant africain ».

Si beaucoup de pays sont impliqués dans le commerce de l'ivoire, certains, comme la République démocratique du Congo, le Nigeria et la Thaïlande, sont considérés comme des plaques tournantes majeures et pérennes de ce trafic. Les réseaux commerciaux asiatiques, opérant à partir de bases africaines, sont maintenant reconnus comme un élément majeur de l'intensification du trafic de l'ivoire.

Le mal persiste avant tout à la base, au sein des États concernés, tant pour ceux qui abritent les éléphants que pour ceux qui participent au commerce illégal de l'ivoire. C'est à ce niveau qu'il devrait être le plus rigoureusement détecté, suivi et combattu, à condition que les gouvernements soient prêts à se mobiliser pour mettre en œuvre les moyens nécessaires, humains et financiers, pour s'opposer au massacre des éléphants et empêcher que le trafic de l'ivoire ne se développe et alimente les réseaux internationaux complexes de la contrebande. Comme le souligne la CITES (2010), la recrudescence du braconnage est avant tout due à une gouvernance inefficace au sein des États.

Les gestionnaires des aires protégées et les institutions impliquées dans la conservation, tout comme le grand public d'ailleurs, manquent d'informations s'agissant des conséquences graves découlant du massacre des éléphants. Quant à ces derniers, ils se comportent comme de véritables " ingénieurs » des écosystèmes de savane ou de forêt (PoILECOT et al., 2007). Modification de la végétation et non obligatoirement « destruction ", transport de graines, interactions avec les autres herbivores, ouverture de milieux fermés au profit d'autres espèces, transformation de la matière végétale, apport de matière organique sont autant d'actions dépendant du pâturage des éléphants et s'inscrivant dans le fonctionnement global des écosystèmes.

Une forte pression de chasse et l'abattage des animaux déstructurent les troupeaux, suppriment les femelles et la mémoire qui guide le groupe, déstabilisent les populations et perturbent la dynamique de l'écosystème.

La mobilisation générale et l'opinion du grand public, qui avaient porté leurs fruits dans les années 1990 pour la conservation de l'éléphant, peuvent constituer un moyen de lutte efficace contre ce trafic mais elles sont insuffisantes. Ce sont les pouvoirs en place, politiques et financiers, qui peuvent favoriser la corruption ou l'enrayer, faciliter la complicité dans un trafic ou la démanteler et donc permettre ou non qu'un système fonctionne bien, dans la légalité et de façon pérenne. 


\section{Références bibliographiques}

ARRANZ L., PFEFFER P., POILECOT P., 2007. Zakouma vingt ans après... Le Courrier de la Nature, 234 : 26-32.

BOUSQUET B., 1986. Projet de conservation du patrimoine naturel en zone Sud du Tchad. Seca/Ministère de l'Environnement et du Tourisme, Tchad.

BOUSQUET B. 1991. Parc national de Zakouma : résultats et inventaires de la faune. Projet « Réhabilitation et conservation du Parc national de Zakouma » ( $\left.n^{\circ} 6.800 .37 .51 .031\right)$. Seca/Ministère de l'Environnement et du Tourisme, Tchad, 27 p. + annexes.

CHARDONNET P., BOULET H., 2008. Des éléphants dans la tourmente, République centrafricaine, 2007. Bois et Forêts des Tropiques, 295 : 91-96.

CITES, 2010. Fifteen Meeting of the Conference of the Parties, Doah (Qatar), 13-25 March 2010. Interpretation and implementation of the convention, species trade and conservation issues : Elephants. Monitoring of illegal hunting in elephant range states. CoP15 Doc. 44.2, 27 p.

DEJACE P., 2002. Zakouma. Ministère de l'Environnement et de l'Eau/Commission européenne, 248 p.

DEJACE P., GAUTIER L., BOUCHE P., 2000. Les populations de grands mammifères et d'autruches du Parc national de Zakouma au Tchad : statuts et tendances évolutives. La Terre et la Vie, 55 : 305-320.

DOLMIA N., 2004. Éléments d'écologie de la population d'éléphants du Parc national de Zakouma. Thèse en sciences de l'environnement, Engref, Montpellier, France, 335 p.

DOLMIA N. M., CALENGE C., MAIILARD D., PLANTON H., 2007. Preliminary observations of elephant (Loxodonta africana Blumenbach) movements and home range in Zakouma National Park, Chad. African Journal of Ecology, 45 : 594-598.
DOUGLAS-HAMILTON I., BURILL A., 1991. Using elephant carcass ratios to determine population trends. In : Kayanja F. I. B., Edroma E. L. (éd.). African Wildlife Research and Management. Paris, France, International Council of Scientific Unions, p. 98-102.

FAY J. M., DOLMIA N. M., BOULANODJI E., NDONINGA A., GUGGEMOS C., POILECOT P., 2005. Comptage aérien total de la grande faune du Parc national de Zakouma. Mee/Curess, Tchad, $35 \mathrm{p}$.

FAY J. M., NGAKOUTOU E. B., TALOUA N., POILECOT P., NDONINGA A., 2006 a. Dénombrement aérien total des grands mammifères et de l'autruche du Parc national de Zakouma, Tchad. Mee/Dcfap/Curess, Tchad, 35 p.

FAY J. M., POILECOT P., N'GAKOUTOU E. B., TOMPKINS D., 2006 b. Dénombrement aérien total des grands mammifères dans le Parc national de Zakouma et sa zone d'influence. Mee/Dcfap/Curess, Tchad, 28 p.

MACKIE C., 2002. Recensement aérien de la grande faune du Parc national de Zakouma. Mee/Dpfpn/ Curess, Tchad, 29 p. + annexes.

MARTIN E., 2005. Large quantities of illegal ivory for sale in Sudan. Kingsfold West Sussex, Royaume-Uni, Care for the Wild International.

MILLIKEN T., BUM R. W., SANGALAKULA L., 2009. The Elephant Trade Information System (ETIS) and the Illicit Trade of Ivory. TRAFFIC Eats/Southern Africa, CoP15 Doc 44.1, Annex, $40 \mathrm{p}$.

NATIONS UNIES, 2005. Profil pays : Tchad. Bureau de la coordination des affaires humanitaires, Bureau régional pour l'Afrique de l'Ouest, $5 \mathrm{p}$.

POILECOT P., 2008. Comptage aérien des grands mammifères (avril 2008) et planification d'une stratégie de suivi écologique au Parc national de Zakouma (Tchad). Rapport final. Agrifor/Eco Consult/Gopa/Curess, 211 p.
POILECOT P., DJIMET B., NGUI T., 2010. La population d'éléphants du parc national de Zakouma, Tchad. Bois et Forêts des Tropiques, sous presse.

POILECOT P., BOULANODJI E., TALOUA N., DJIMET B., NGUI T., SINGA J., 2007. Parc national de Zakouma : des éléphants et des arbres. Bois et Forêts des Tropiques, 291 (1) : 13-24.

POTGIETER D., TALOUA N., DJIMET B., FAY M., 2009. Dry season aerial total count, Zakouma national Park, Chad. Wcs/UE/Curess, 27 p.

STILES D., MARTIN E., 2009. The USA's ivory market. How much a threat to elephants ? Pachyderm, 45 : 67-76.

WASSER K. L., WILLIAM W. J., DRORI O., KISAMO E. S., MAILAND C., MUTAYOBA B., MATTHEW STEPHENS M., 2008. Combating the illegal trade in african elephant ivory with DNA forensics. Conservation Biology, 22 (4) : 1065-1071.

WASSER S., CLARK B., LAURIE C., 2009. Sur la piste de l'ivoire. Pour la Science, 383 : 24-30. 